Results Awareness: Over 40\% of patients and carers stated they did not know that weight loss, tiredness and chest pain are possible symptoms of lung cancer.

Screening: 52\% of HCPs believe their Government should introduce a national screening programme for lung cancer.

Early diagnosis: 68\% of HCPs believe that prompt access to investigative testing including blood tests, chest x-rays and CT scans are the greatest challenge to rapid diagnosis.

Waiting times: $36 \%$ of patients were waiting over one month for confirmation of a suspected lung cancer diagnosis and 17\% waited over two months.

Achievability of survival: $49 \%$ of patients and carers believe surviving lung cancer for more than five years to be achievable. However, $65 \%$ of HCPs consider it difficult to achieve and $15 \%$ "completely unachievable".

Local variation: $84 \%$ of the HCPs believe regional inequalities in health and care services have a moderate or major impact on lung cancer survival rates.

Conclusion Analysis of the results shows that improvement in prevention, early-stage diagnosis and awareness of signs and symptoms among the public, could play a significant part in improving five-year survival. The results also show that patients and carers are more optimistic about the achievability of surviving lung cancer for more than five years (though this discounts patients who were not well enough to complete the survey) than HCPs.

\section{P107 PREDICTORS OF MORTALITY IN PATIENTS UNDERGOING LUNG CANCER SURGERY}

${ }^{1} \mathrm{H}$ Law, ${ }^{1} \mathrm{P}$ Foden, ${ }^{1} \mathrm{M}$ Evison, ${ }^{2} \mathrm{~F}$ Wallace, ${ }^{2} \mathrm{~A}$ Ashworth, ${ }^{3} \mathrm{R}$ Shah, ${ }^{1} \mathrm{P}$ Crosbie, ${ }^{1} \mathrm{R}$ Booton. ${ }^{1}$ North West Lung Centre, UHSM, Manchester, UK; ${ }^{2}$ Cardiothoracic ICU, UHSM, Manchester, UK; ${ }^{3}$ Department of Cardiothoracic Surgery, UHSM, Manchester, UK

\subsection{6/thoraxjnl-2016-209333.250}

Introduction Surgical resection is a treatment of choice for patients with early stage lung cancer and physiological measurements are routinely used to help predict post-operative risk, particularly in the 'high-risk' patient group.

At present, there is a lack of concordance between current available guidelines incorporating the use of such parameters aimed to guide decisions on surgery for high-risk patients with lung cancer. As a result, the decision to operate will differ for a particular patient depending on which guidelines are consulted.

We aim to identify which parameters best predicts post-operative mortality and whether this information can be used to construct a more encompassing pre-operative risk prediction model to help guide these difficult decision processes.

Methods Retrospective analysis of all patients undergoing CPET (cardio-pulmonary exercise testing) prior to lung cancer surgery between 01/01/2012 and 31/12/2015 was carried out. Age, BMI along with pre-operative and post-operative predicted physiological parameters were reviewed and statistical analysis performed. We also looked at survival based on type of surgery (sub-lobar, lobar, pneumonectomy), histology and cancer staging.

Results Single variable analysis of the 178 patients identified that low BMI $(\mathrm{p}=0.005)$ and PPO DLCO $\%(\mathrm{p}=0.004)$ were associated with greater post-operative mortality risk.

There was a statistically significant difference between different cancer stage and type of surgery as expected.
Using the probabilities from the logistical regression model to predict one-year mortality gives an AUC of 0.764. A probability cut-off of 0.167 used to predict whether a patient will die within one year of surgery provides a sensitivity of $76.5 \%$, specificity 66.4\%, PPV 35.1\% and NPV 92.2\%.

Conclusions Contrary to current guidelines, CPET data did not seem to carry statistically significant weighting in determining post-operative mortality outcomes in our patient group with BMI and PPO DLCO\% showing a stronger, statistically significant association.

Absolute\% change between pre and PPO FEV1 values appears to be a good predictor of one-year mortality following surgery.

Further work is required but early analysis suggested that parameters such as BMI, PPO DLCO\% and absolute post-operative change in $\mathrm{FEV} 1 \%$ can be used to construct a pre-surgical prediction model for 'high-risk' patients undergoing surgery for lung cancer.

\begin{tabular}{llll}
\multicolumn{4}{l}{ Abstract P107 Table 1} \\
\hline Variable & $\mathbf{n}$ & Hazard ratio $(95 \%$ Cl) & Cox regression p-value \\
\hline Age & 178 & $0.988(0.957,1.020)$ & 0.47 \\
BMI & 178 & $0.932(0.887,0.979)$ & 0.005 \\
FEV1\% predicted (pre) & 178 & $1.008(0.999,1.018)$ & 0.088 \\
FEV1\% predicted (post) & 177 & $0.998(0.987,1.010)$ & 0.73 \\
DLCO\% predicted (pre)* & 137 & $0.994(0.976,1.013)$ & 0.54 \\
DLCO\% predicted (post) & 136 & $0.963(0.939,0.988)$ & 0.004 \\
V02 max & 178 & $1.035(0.975,1.098)$ & 0.26 \\
V02 max\% predicted (pre) & 178 & $1.003(0.991,1.015)$ & 0.66 \\
V02 max\% predicted (post) & 177 & $0.990(0.977,1.003)$ & 0.14 \\
VEC02 & 176 & $1.024(0.996,1.053)$ & 0.091 \\
\hline
\end{tabular}

\section{P108 LUNG CANCER RESECTION OUTCOMES IN THE FIRST YEAR: A 5 YEAR REVIEW}

J Ibrahim, A Mukhopadhyay, V Lostarakos, A Mahmood, S Khan, N Maddekar, I Hussain, A Lea, C Satur, Q Abid, S Ghosh, M Haris. Royal Stoke University Hospital, Stoke-on-trent, UK

\subsection{6/thoraxjnl-2016-209333.251}

Introduction Lung cancer survival remains poor despite the use of advanced diagnostic and therapeutic interventions. Surgical resection offers the best chance of cure for those with early stage lung cancer. Those who undergo curative resection for non-small cell lung cancer remain at risk of recurrence. We wished to evaluate thoracotomy outcomes in patients undergoing resection with curative intent.

Methods Retrospective review of consecutive surgical resections for suspected or confirmed lung cancer over 5-year period between January 2010 and December 2014 in a tertiary thoracic unit. Patients referred from other centres were excluded. Surgical database and post-operative follow up information was reviewed. Futile thoracotomies were defined as inoperable lung cancer at the time of surgery, benign lung lesion, incomplete tumour resection margins and recurrence or death in the first year. ${ }^{1}$

Results 298 patients underwent surgical resection; mean age 68 years (range 26-91); male 150 (50\%). 48 (16\%) had histo-cytological confirmation of lung cancer prior to resection. Post-operative histology revealed 248 (83\%) primary lung cancer; 31 (10\%) benign pathology; 17 (6\%) metastatic tumour from other 